\title{
Traços de personalidade de adolescentes infratores e vitimizados por meio do Eysenck Personality Questionnaire Junior (EPQ-J)
}

\author{
Antonio Augusto Pinto Junior* \\ Leila Salomão de La Plata Cury Tardivo** \\ Helena Rinaldi Rosa ** \\ Gilsaine Chaves** \\ Gabriel Okawa Belizário***
}

\begin{abstract}
Resumo
O presente estudo objetivou mapear os traços de personalidade (Psicoticismo, Neuroticismo e Extroversão) de adolescentes brasileiros vítimas de abuso intrafamiliar, nas modalidades física e/ou sexual, e autores de ato infracional. A amostra foi composta por um total de 200 adolescentes de ambos os sexos na faixa etária entre 12 e 16 anos, divididos em três grupos: a) 50 adolescentes infratores, b) 50 adolescentes vítimas de abuso intrafamiliar físico e/ou sexual, c) 100 adolescentes sem suspeitas de sofrerem violência doméstica ou terem praticado ato infracional. Como instrumento, foi utilizado o Eysenck Personality Questionnaire Junior (EPQ-J). Os dados foram avaliados pela análise da significância da diferença entre os grupos de pesquisa por meio do teste de comparação múltipla de Tukey. Foram encontradas diferenças significantes entre os grupos de pesquisa nos tres fatores analisados (Psicotisismo $\mathrm{p}<0,001$; Extroversão $\mathrm{p}<0,015$; Neuroticismo $\mathrm{p}<0,027$ ), indicando principalmente nos grupos clínicos tendência a reagir de forma desajustada, impulsiva e agressiva. Também se mostraram mais introvertidos, não sociáveis, com isolamento social, além de apresentarem hipersensibilidade emocional, com excitabilidade e traços de ansiedade, que podem desencadear transtornos de humor, principalmente a depressão. Conclui-se que as experiências de vitimização intrafamiliar e de prática de atos infracionais, além de se mostrarem como faces de uma mesma moeda, prejudicam a adaptação do indivíduo e interferem na forma como atuam no ambiente, podendo levar ao comprometimento da saúde mental de sujeitos e ao desencadeamento de transtornos de humor ou de carater.
\end{abstract}

Palavras-chave: adolescência, traços de personalidade, violência.

\section{Abstract}

The present study aimed to map the personality traits (Psychoticism, Neuroticism and Extroversion) of Brazilian adolescents victims of intrafamilial abuse, in physical and/or sexual modalities, and perpetrators of an infrational act. The sample consisted of a total of 200 adolescents of both sexes aged 12 to 16 years, divided into three groups: a) 50 young offenders, b) 50 adolescents victims of physical and/or sexual intrafamilial abuse, c) 100 adolescents who are not suspected of experiencing domestic violence or have committed an infraction act. As an instrument, the Eysenck Personality Questionnaire Junior (EPQ-J) was used. Data were evaluated by analyzing the significance of the difference between the research groups using Tukey's multiple comparison test. Significant differences were found between the research groups in the three analyzed factors (Psychoticism p <0.001; Extroversion p <0.015; Neuroticism $\mathrm{p}<0.027)$, indicating mainly in the clinical groups a tendency to react inappropriately, impulsively and aggressively. They were also more introvert, non-sociable, with social isolation, besides presenting emotional hypersensitivity, excitability and anxiety traits, which can trigger mood disorders, especially depression. It is concluded that the experiences of intrafamilial abuse and the practice of infraction act, besides being shown as two sides of the same coin, impair the individual's adaptation and interfere the way they act in the environment, which may lead to impairment of the mental health of subjects. and triggering mood or character disorders.

Key-words: adolescence, personality traits, violence.

* Professor associado da Universidade Federal Fluminense

**Professora Associada Universidade de São Paulo

***Mestre em Psicologia Clínica pela Universidade de São Paulo 


\section{Introdução}

A violência não é marca registrada de nossa sociedade contemporânea. Ela está inscrita na existência humana desde seus primórdios. A cada época ela se manifesta de formas e em contextos diferentes. Contudo, seu incremento na atualidade configura-se como aspecto representativo e problemático da organização da vida social, sendo considerada uma das principais causas de morte de pessoas entre 15 e 44 anos em todo o mundo, além das inúmeras sequelas biopsicossociais em nível individual e coletivo (DeVerteuil, 2015; Littman \& Paluck, 2015).

$\mathrm{O}$ fenômeno da violência, em sua complexidade e multicausalidade, tem atingido também a adolescência. Os adolescentes, ao se envolverem com a violência, seja na condição de vítimas ou na de infratores, são expostos a alguma forma de exclusão e/ou sofrimento. Na condição de vítima, verifica-se a vivência de situações abusivas e de opressão (Cook, Spinazzola, Ford, Lanktree, Blaustein, Cloitre \& Mallah, 2017). Na situação de agressor, o jovem é excluído da possibilidade de viver o exercício da cidadania, por meio da qual pode reconhecer-se e ser reconhecido como sujeito de direitos e deveres (Chen, Voisin \& Jacobson, 2016). Estudos mostram a existência de correlação positiva entre a experiência de vitimização doméstica na infância e a manifestação de comportamentos violentos e/ou disruptivos na adolescência, indicando que o ser vítima e o ser vitimizador representam duas faces de uma mesma moeda (Cook et al, 2017; Mrug, Madan \& Windle, 2016; Finkelhor, Turner, Shattuck, Hamby \& Kracke, 2015).

A adolescência é uma fase do desenvolvimento humano que implica mudanças e conflitos que podem, se não bem manejados, levar os jovens a níveis de instabilidade ou a erupção de distúrbios emocionais (Evans, 2015). A exposição à violência intrafamiliar ou comunitária aumenta consideravelmente esse risco e é responsável por inúmeros agravos à saúde mental de crianças e adolescentes. Muitas sequelas têm sido identificadas, tais como: ansiedade, depressão, reprodução da violência sofrida e outros quadros psicopatológicos (Heleniak, Jenness, Vander Stoep, McCauley \& McLaughlin, 2016; Busso, McLaughlin \& Sheridan, 2017).

Dessa forma, torna-se fundamental o investimento em pesquisas na área da avaliação psicológica com instrumentos que mapeiem os aspectos emocionais e de personalidade dos adolescentes expostos à violência (seja na condição de vítimas ou na de infrator), pois com tais informações é possível o desenvolvimento de estratégias interventivas e terapêticas mais eficientes para cada caso. É importante ressaltar que a avaliação psicológica é definida como um processo de coleta, investigação e interpretação de dados, no qual se conhece o avaliado e sua demanda, com o objetivo de programar a tomada de decisão mais apropriada do professional de saúde mental. Deve-se também destacar que esse processo envolve diferentes técnicas e procedimentos, tais como a entrevista e os testes psicológicos. Esses últimos são tradicionalmente classificados e diferenciados de acordo com sua objetividade e padronização, principalmente os testes psicométricos e as escalas. Em relação aos testes psicométricos, esses buscam mensurar por meio de critérios objetivos o atributo ou construto que estão avaliando (p. ex., quociente de inteligência e fatores de personalidade). (Loewenthal \& Lewis 2018).

Dentre os testes psicométricos de personalidade, especialmente desenvolvidos para adolescentes, destaca-se o Questionário de Personalidade para Crianças e Adolescentes (EPQ-J), que condensa os resultados dos estudos do psicólogo inglês Hans Eysenck (Eysenck e Eysenck, 2013) e está baseado em seu modelo PEN de personalidade, que compreende três grandes fatores Psicoticismo, Extroversão e Neuroticismo. Os autores definiram as referidas dimensões, fundamentais para a personalidade humana, como:

Psicoticismo: altas pontuações indicam pessoas agressivas, frias, egocêntricas, impessoais, impulsivas, antissociais, não-empáticas, criativas e obstinadas. Já, indivíduos com escores baixos nesse fator apresentam características contrárias e são definidos pelo controle de impulsos. Neuroticismo: sujeitos com altos escores neste fator são definidos como ansiosos, deprimidos, tensos, irracionais, tímidos, melancólicos, emotivos, com tendência a sentir culpa e baixa autoestima. Por outro lado, baixos escores nesse fator caracterizam o indivíduo como emocionalmente estável. Por fim, o fator de Extroversão, no qual altos escores descrevem a pessoa como sociável, animada, ativa, assertiva, despreocupada, dominante, cordial e aventureira. Baixos escores reúnem características opostas e definem o sujeito como introvertido.

A versão brasileira desse teste, traduzida e adaptada por Flores-Mendoza (2013), tem 60 itens, sendo 18 de Neuroticismo, 12 de Extroversão e 14 de Psicoticismo, e ainda uma quarta escala denominada Sinceridade (elaborada a fim de avaliar participantes que podem ser menos sinceros em suas respostas ao questionário).

O presente estudo objetivou mapear as características de personalidade (Psicoticismo, Neuroticismo e Extroversão) de adolescentes brasileiros vítimas de vio- 
lência intrafamiliar ou envolvidos com atos infracionais por meio da aplicação do Questionário de Personalidade para Crianças e Adolescentes (EPQ-J), pois os resultados podem fundamentar projetos de intervenção clínica e de prevenção do abuso infantil em nossa realidade.

\section{Método}

\section{Participantes}

A amostra foi composta por um total de 200 adolescentes brasileiros dos estados de São Paulo e do Rio de Janeiro, na faixa etária entre 12 e 16 anos, divididos em três grupos: a) Grupo de Adolescentes Infratores, composto por 50 adolescentes de ambos os sexos cumprindo medida socioeducativa por prática e ato infracional em instituições especializadas para tal; b) Grupo de adolescentes vítimas de violência intrafamiliar, composto por 50 adolescentes, de ambos os sexos, comprovadamente vítimas de alguma modalidade de violência, física e/ou sexual, praticada pelos pais ou responsáveis, atendidos em instituição de assistência social; e c) Grupo Controle, composto por 100 adolescentes, de ambos os sexos sem suspeitas de sofrerem violência doméstica ou terem praticado ato infracional, selecionados em escolas públicas. Na Tabela 1 é apresentada a distribuição dos participantes quanto ao sexo e a idade. Para avaliar se a distribuição percentual dos participantes era a mesma entre os grupos, utilizou-se o Teste Qui-Quadrado de Pearson para obter os valores de p do teste. Para a análise da Escolaridade utilizou-se a Análise de Variância Anova, descrevendo os resultados em Média e Desvio Padrão.

\section{Procedimentos}

A pesquisa foi avaliada e aprovada pelo Comitê de ética em Pesquisa da Faculdade de Medicina da universidade Federal Fluminense (FM/UFF/HU), sob o protocolo $\mathrm{n}^{\circ}$ 1.425.412. O EPQ-J were aplicado e respondido by all participants from três research groups. The data of the present study was collected individually. All participants and parents/legal guardians signed an informed consent form.

\section{Análise dos Dados}

A avaliação do EPQJ de cada participante foi realizada segundo as orientações do manual apresentado por Flores-Mendoza (2013), para as quatro escalas avaliadas por esse instrumento (Psicoticismo, Extroversão; Neuroticismo e Sinceridade). As respostas de cada participante foram expressas em dados numéricos $(1=\operatorname{Sim} ; 0=$ Não $)$ e transcritas para uma planilha de Excel para permitir que fossem realizadas comparações entre os grupos clínicos e de controle. Para avaliar a significância da diferença entre os grupos e a comparação do grupo Controle com o de adolescentes infratores e com o de vitimizados foi utilizado o teste de Comparação múltipla de Tukey.

\section{Resultados e discussão}

Ao se analisar a idade dos participantes, percebe-se os grupos controle e de vítimas de violência intrafamiliar

Tabela 1. Distribuição dos participantes quanto a Sexo, Idade e Escolaridade.

\begin{tabular}{ll|ccc|c}
\hline Característica & Grupo & Controle & $\begin{array}{c}\text { Adolescente } \\
\text { Infrator }\end{array}$ & $\begin{array}{c}\text { Violência } \\
\text { Doméstica }\end{array}$ & $\begin{array}{c}\text { Valor de } \\
\mathrm{p}\end{array}$ \\
\hline 12 & $12(12,0 \%)$ & $(0,0 \%)$ & $8(16,0 \%)$ & $\mathbf{0 , 0 0 0 2}$ \\
& 13 & $13(13,0 \%)$ & $2(4,0 \%)$ & $13(26,0 \%)$ & \\
& 14 & $13(13,0 \%)$ & $4(8,0 \%)$ & $7(14,0 \%)$ & \\
& 15 & $18(18,0 \%)$ & $6(12,0 \%)$ & $8(16,0 \%)$ & \\
& 16 & $44(44,0 \%)$ & $38(76,0 \%)$ & $14(28,0 \%)$ & \\
\hline Sexo & Feminino & $54(54,0 \%)$ & $10(20,0 \%)$ & $27(54,0 \%)$ & $\mathbf{0 , 0 0 0 2}$ \\
& Masculino & $46(46,0 \%)$ & $40(80,0 \%)$ & $23(46,0 \%)$ & \\
\hline Escolaridade* & & $9,58(1,65)$ & $7,84(2,48)$ & $6,84(2,06)$ & $<\mathbf{0 , 0 0 1}$ \\
\hline Total Geral & & $100(100,0 \%)$ & $50(100,0 \%)$ & $50(100,0 \%)$ & \\
\hline
\end{tabular}

*Resultados expressos em Média (Desvio Padrão) - ANOVA 
com percentuais muito próximos, sendo o último com um percentual um pouco maior na faixa dos 13 e 16 anos.

A distribuição mais equilibrada entre as diferentes faixas etárias principalmente no grupo de vítimas deve-se ao fato amplamente destacado pela literatura sobre maus-tratos na infância e adolescência acerca das características das vítimas no que se refere à idade. Os estudos mostram que o abuso infantil é um fenômeno que atinge vítimas de diferentes faixas etárias indiscriminadamente (Bottoms, Peter-Hagene, Epstein, Wiley, Reynolds, \& Rudnicki, 2016; Kloppen, Haugland, Svedin, Mæhle, \& Breivik, 2016; Aydin, Akbas, Turla, Dundar, Yuce, \& Karabekiroglu, 2015).

Por outro lado, verifica-se que o grupo de adolescents infratores apresenta maiores diferenças, tendo a concentração maior na faixa dos 16 anos, com diferença significante (valor de $\mathrm{p}=0.0002$ ). Esses dados corroboram os achados da literatura na área que indicam que a maioria dos adolescentes cumprindo medidas socioeducativas em função da prática de atos infracionais se concentra majoritariamente na faixa etária entre 15 e 17 anos (Wolff, Baglivio, \& Piquero, 2017; Weng, Ran, \& Chui, 2016; Baglivio, Wolff, Piquero, \& Epps, 2015).

A análise da variável sexo mostra os grupos controle e de vitimizados bem semelhantes e o grupo de infratores com muito mais participantes do sexo masculino, apresentando diferença significante (valor de $\mathrm{p}=0.0002$ ). Essa caracterização das amostras no quesito sexo também confirma os resultados dos estudos sobre o perfil de adolescentes infratores que apontam que a maioria deles é do sexo masculino ((Wolff, Baglivio \& Piquero, 2017; Weng, Ran \& Chui, 2016; Baglivio, Wolff, Piquero \& Epps, 2015). Em contrapartida, a literatura tem indicado que a distribuição de vítimas de abuso intrafamiliar em termos de sexo é mais igualitária (Bottoms, et. Al. 2016; Kloppen, Haugland, Svedin, Mæhle \& Breivik, 2016; Aydin, Akbas, Turla, Dundar, Yuce \& Karabekiroglu, 2015).

Quanto à escolaridade, os sujeitos do grupo controle apresentam mais anos de estudo que os participantes dos grupos clínicos dessa pesquisa. Tal diferença significante entre os três grupos também é respaldada pela literatura científica nesse campo do conhecimento. Pesquisas com adolescentes em conflito com a lei sinalizam que o nível educacional desses jovens é sempre identificado como inferior, sendo frequente o abandono escolar (Fine, Simmons, Miltimore, Steinberg, Frick \& Cauffman, 2018; Ortega-Campos, García-García, Gil-Fenoy, \& Zaldívar-Basurto, 2016). Da mesma forma, vítimas de violência intrafamiliar comumente mostram, segundo a literatura, dificuldades de aprendizagem, atraso e abandono escolar em função das diversas consequências da vitimização sofrida que afetam o desenvolvimento cognitivo, intelectual e social das vítimas. Alguns estudos destacam, ainda, que a presença de dificuldades graves de aprendizagem pode ser considerada, junto com outros sinais e sintomas específicos, um indicador importante de vitimização doméstica (Waal, Dekker, Kikkert, Kleinhesselink \& Goudriaan, 2017; Davis, Moss, Nogin, \& Webb, 2015).

Os resultados da comparação entre os grupos Controle, adolescents infratores e vitimizados de acordo com os escores do EPQJ são apresentados na Tabela 2, e os dados expressos em Média (Desvio Padrão) - ANOVA.

$\mathrm{Na}$ análise dess resultados se constata diferenças significativas nas três dimensões avaliadas. Em relação ao Psicoticismo, o grupo controle apresentou os resultados mais baixos, seguido do grupo de infratores, e depois do grupo de vitimizados, com a maior média. De acordo com Flores-Mendoza (2013) sujeitos com escores mais altos nessa dimensão costumam agir com frieza, desajuste, impulso e agressividade. Dessa forma, destaca-se uma tendência dos adolescentes dos grupos clínicos (infratores e vítimas) a agirem por impulso, sem consideração ao que pensa ou sente o outro. Essa constatação pode estar relacionada com as consequências das vivências infantis de maus tratos, violência e negligência pelas figuras parentais (Heleniak, Jenness, Vander Stoep, McCauley, \& McLaughlin, 2016; Busso, McLaughlin, \& Sheridan, 2017; Cook et al, 2017). Especialmente, em relação aos adolescents infratores, os estudos demonstram que traços de insensibilidade e

Tabela 2. Comparação entre os grupos nos escores do EPQJ

\begin{tabular}{cl|l|l|l|l}
\hline \multicolumn{1}{l|}{ Fatores } & \multicolumn{1}{|c|}{$\begin{array}{c}\text { Infratores } \\
(\mathbf{n}=\mathbf{5 0})\end{array}$} & \multicolumn{1}{|c|}{$\begin{array}{c}\text { Vítimas } \\
\mathbf{( n = 5 0 )}\end{array}$} & \multicolumn{1}{|c}{$\begin{array}{c}\text { Controle } \\
\mathbf{( n = 1 0 0 )}\end{array}$} & valor de $\mathbf{p}$ \\
\hline EPQJ & & & & & \\
- & Psicoticismo & $3,60(2,70)$ & $3,84(3,25)$ & $2,16(1,84)$ & $<\mathbf{0 , 0 0 1}$ \\
- Extroversão & $7,96(2,67)$ & $8,86(2,25)$ & $9,23(2,52)$ & $\mathbf{0 , 0 1 5}$ \\
- Neuroticismo & $9,18(3,93)$ & $10,50(4,69)$ & $11,36(4,95)$ & $\mathbf{0 , 0 2 7}$ \\
- Sinceridade & $10,26(3,26)$ & $9,32(4,44)$ & $9,95(3,56)$ & 0,435 \\
\hline
\end{tabular}


impulsividade apresentam associação significativa com o comportamento infracional, principalmente para os adolescentes vivendo em áreas de maior vulnerabilidade social (Chen, Voisin \& Jacobson, 2016; Weng, Ran \& Chui, 2016).

No que se refere à dimensão Extroversão, verifica-se que o grupo controle obteve a maior pontuação, seguido do grupo de vítimas e por último o grupo de infratores. Para Flores-Mendoza (2013) sujeitos com escores mais altos nessa categoria tendem a se mostrar mais extrovertidos, caracterizados pela sociabilidade, vivacidade, otimismo e outros traços. Em contrapartida, sujeitos com escores mais baixos mostram-se mais introvertidos e são caracterizados como quietos, não sociáveis, reservados, pessimistas e controlados. Por essa interpretação, encontra-se coerência com traços de personalidade que são apresentados tanto pelo grupo de infratores quanto pelo de vítimas, em função das consequências das experiências traumáticas vividas e pela constatação do modo de atuar, principalmente dos adolescentes infratores, não respeitando as regras sociais (Heleniak, Jenness, Vander Stoep, McCauley, \& McLaughlin, 2016; Busso, McLaughlin, \& Sheridan, 2017; Cook et al, 2017). Diante desses dados, pode-se inferir que as experiências traumáticas do abuso intrafamiliar incrementam ou favorecem o isolamento social, que é um dos aspectos comportamentais presentes nos grupos clínicos dessa pesquisa.

Ao analisar a categoria Neurotiscismo, os dados apontam que o grupo controle mostrou a pontuação maior, seguido do grupo de vitimizados, e depois do grupo de infratores. Flores-Mendoza (2013) assinala que escores mais altos nessa categoria indicam hipersensibilidade emocional, com excitabilidade e traços de ansiedade. Há um consenso na literatura de que a presença substancial de neuroticismo no sujeito é um fator de risco para o desenvolvimento de transtornos mentais na vida adulta, principalmente a depressão (Aldinger, 2014; Andrés, Richaud de Minzi, Castañeiras, Canet-Juric \& Rodríguez-Carvajal, 2016). A presença de maior valor no controle deve ser observada com cautela. Ainda que as diferenças sejam significantes, pode-se observar que todas as médias estão dentro da faixa da normalidade segundo a tabela normativa do Manual brasileiro (Eysenck \& Eysenck, 2013).

Um índice mais elevado de neuroticismo no grupo de vítimas de violência intrafamiliar pode ser considerado uma variável que pode se associar a outros fatores comuns das sequelas do abuso e potencializar uma problemática psicopatológica mais tarde na vida adulta.
Já na análise da categoria Sinceridade no EPQJ nota-se os três grupos muito próximos, destacando o grupo de vítimas com a menor média. Nesse item não foram encontradas diferenças significativas entre o grupo controle e os grupos clínicos, permanecendo todos na média estipulada pelo teste, o que denota que os respondentes, no geral, não agiram de forma tendenciosa para passar uma boa imagem de si, e que os resultados se mostram confiáveis.

\section{Conclusões}

A vivência e exposição à violência na infância e na adolescência têm sido reconhecidas como um grave problema de saúde pública, o que se deve à sua alta incidência mundialmente e às consequências e sequelas que causam no desenvolvimento psicossocial e na saúde mental das vítimas. Essa situação exige o desenvolvimento de políticas de prevenção, visando combater esse fenômeno. Além disso, é importante investir em pesquisas com foco no mapeamento dos traços de personalidade de adolescentes envolvidos em situações de abuso intrafamiliar e em práticas delituosas para que estratégias interventivas e de tratamento possam ser aprimoradas.

Diante dos resultados da presente pesquisa, verifica-se que os fatores avaliados pelo EPQJ, ou seja, Psicoticismo, Extroversão e Neuroticismo, na amostra de adolescentes vitimizados e infratores em comparação com adolescentes sem essas condições, apontam para o comprometimento da saúde mental de sujeitos que estão ou que foram expostos à violência intrafamiliar. Dessa forma, ser vítima de abuso ou cometer atos infracionais, além de se mostrarem como faces de uma mesma moeda, denotam que essas experiências prejudicam a adaptação do indivíduo e interferem na forma como atuam no ambiente. Constatou-se que os grupos clínicos dessa investigação tendem a reagir mais de forma desajustada, impulsiva e agressiva, desconsiderando o que pensa ou sente o outro, o que pode trazer graves consequência futuras, principalmente em termos do desenvolvimento de uma conduta antisocial ou psicopática (fator P). Da mesma forma, constatou-se que os adolescentes dos grupos clínicos se revelaram muito mais introvertidos, não sociáveis, tendendo ao isolamento social, o que pode levá-los a manifestar sérios problemas no campo da interação grupal e cumprimento das leis sociais (Fator E). Além disso, encontrou-se no grupo de vítimas de violência intrafamiliar uma tendência à hipersensibilidade emocional, com excitabilidade e traços de ansiedade (Fator $\mathrm{N}$ ), que podem culminar na eclosão de transtornos 
de humor, principalmente a depressão, como bem aponta a literatura na área da vitimização infantil.

De forma geral, apesar de se delinear características importantes acerca dos fatores de personalidade em adolescentes envolvidos ou vítimas de violência, por se tratar de um estudo transversal, há limitações inerentes ao método utilizado, pois a amostra não foi aleatória, dependendo da permissão dos pais para a participação, o que também pode trazer algumas restrições à generalização dos resultados para a população geral. No entanto, o tamanho da amostra aqui pesquisada oferece alguma confiança na generalização de conclusões. Uma possibilidade futura de investigação seria um projeto de pesquisa longitudinal para verificar se, ao longo do tempo aqueles indivíduos com traços aqui detectados mostrariam maior tendência a desenvolver um quadro psicopatológico mais grave. De qualquer forma, é importante entender que estratégias de prevenção devem ser sempre priorizadas para que se possa evitar possíveis traumas que interrompam o desenvolvimento psicossocial de crianças e adolescentes, e que, quando se constata a vitimização nessa parcela da população, investigações com diferentes estratégias interventivas devem ser conduzidas visando avaliar aquelas que podem reverter a direção das relações entre esses fatores.

\section{Referências}

Aldinger, M., Stopsack, M., Ulrich, I., Appel, K., Reinelt, E., Wolff, S., ... \& Barnow, S. (2014). Neuroticism developmental courses-implications for depression, anxiety and everyday emotional experience; a prospective study from adolescence to young adulthood. BMC Psychiatry, 14(1), 210. doi: 10.1186/s12888-014-0210-2

Andrés, M. L., Richaud de Minzi, M. C., Castañeiras, C., Canet-Juric, L., \& Rodríguez-Carvajal, R. (2016). Neuroticism and depression in children: the role of cognitive emotion regulation strategies. The Journal of Genetic Psychology, 177(2), 55-71. doi: 10.1080/00221325.2016.1148659

Aydin, B., Akbas, S., Turla, A., Dundar, C., Yuce, M., \& Karabekiroglu, K. (2015). Child sexual abuse in Turkey: an analysis of 1002 cases. Journal of Forensic Sciences, 60(1), 61-65. doi: 10.1111/1556-4029.12566

Baglivio, M. T., Wolff, K. T., Piquero, A. R., \& Epps, N. (2015). The relationship between adverse childhood experiences (ACE) and juvenile offending trajectories in a juvenile offender sample. Journal of Criminal Justice, 43(3), 229-241. doi: 10.1016/j.jcrimjus.2015.04.012

Bottoms, B. L., Peter-Hagene, L. C., Epstein, M. A., Wiley, T. R., Reynolds, C. E., \& Rudnicki, A. G. (2016). Abuse characteristics and individual differences related to disclosing childhood sexual, physical, and emotional abuse and witnessed domestic violence. Journal of Interpersonal Violence, 31(7), 1308-1339.

Busso, D. S., McLaughlin, K. A., \& Sheridan, M. A. (2017). Dimensions of adversity, physiological reactivity, and externalizing psychopathology in adolescence: Deprivation and threat. Psychosomatic Medicine, 79(2), 162. doi: 10.1097/PSY.0000000000000369
Chen, P., Voisin, D. R., \& Jacobson, K. C. (2016). Community violence exposure and adolescent delinquency: examining a spectrum of promotive factors. Youth \& Society, 48(1), 33-57. https://doi.org/10.1177/0044118X13475827

Cook, A., Spinazzola, J., Ford, J., Lanktree, C., Blaustein, M., Cloitre, M. \& Mallah, K. (2017). Complex trauma in children and adolescents. Psychiatric Annals, 35(5), 390-398. doi: 10.3928/00485713-20050501-05

Davis, A. S., Moss, L. E., Nogin, M. M., \& Webb, N. E. (2015). Neuropsychology of child maltreatment and implications for school psychologists. Psychology in the Schools, 52(1), 77-91. doi: 10.1002/pits.21806

DeVerteuil, G. (2015). Conceptualizing violence for health and medical geography. Social Science \& Medicine, 133, 216-222. doi: 10.1016/j.socscimed.2015.01.018

Evans, J. H. (2015). Adolescent and pre-adolescent psychiatry. New York NC: Academic Press.

Eysenck, H. J., \& Eysenck, S. B. G. (2013). Questionário de Personalidade para Crianças e Adolescentes (EPQ-J). São Paulo BR: Vetor.

Fine, A., Simmons, C., Miltimore, S., Steinberg, L., Frick, P. J., \& Cauffman, E. (2018). The school experiences of male adolescent offenders: Implications for academic performance and recidivism. Crime \& Delinquency, 64(10), 1326-1350. doi: 10.1177/0011128717750392

Finkelhor, D., Turner, H., Shattuck, A., Hamby, S., \& Kracke, K. (2015). Children's exposure to violence, crime, and abuse: an update. Washington, DC: US Department of Justice, Office of Justice Programs, Office of Juvenile Justice and Delinquency Prevention.

Flores-Mendoza, C. (2013). Estudo brasileiro do EPQ-J (Adaptação e Validação). In Eysenck, H. J.; Eysenck, S. B. G. Questionário de Personalidade para Crianças e Adolescentes (EPQ-J). São Paulo: Vetor.

Heleniak, C., Jenness, J. L., Vander, Stoep, A., McCauley, E., \& McLaughlin, K. A. (2016). Childhood maltreatment exposure and disruptions in emotion regulation: a transdiagnostic pathway to adolescent internalizing and externalizing psychopathology. Cognitive Therapy and Research, 40(3), 394-415. doi: 10.1007/s10608-015-9735-z

Kloppen, K., Haugland, S., Svedin, C. G., Mæhle, M., \& Breivik, K. (2016). Prevalence of child sexual abuse in the Nordic countries: a literature review. Journal of Child Sexual Abuse, 25(1), 37-55. doi: 10.1080/10538712.2015.1108944

Littman, R., \& Paluck, E. L. (2015). The cycle of violence: Understanding individual participation in collective violence. Political Psychology, 36, 79-99. doi: $10.1111 /$ pops.12239

Loewenthal, K., \& Lewis, C. A. (2018). An introduction to psychological tests and scales. London UK: Psychology Press.

Mrug, S., Madan, A., \& Windle, M. (2016). Emotional desensitization to violence contributes to adolescents' violent behavior. Journal of Abnormal Child Psychology, 44(1), 75-86. doi: 10.1007/s10802-015-9986-x

Ortega-Campos, E., García-García, J., Gil-Fenoy, M. J., \& Zaldívar-Basurto, F. (2016). Identifying Risk and protective factors in recidivist juvenile offenders: a decision tree approach. Plos One, 11(9), e0160423. doi: 10.1371/ journal.pone.0160423

Waal, M. M., Dekker, J. J., Kikkert, M. J., Kleinhesselink, M. D., \& Goudriaan, A. E. (2017). Gender differences in characteristics of physical and sexual victimization in patients with dual diagnosis: a cross-sectional study. BMC Psychiatry, 17(1), 270. doi: 10.1186/s12888-017-1413-0

Weng, X., Ran, M. S., \& Chui, W. H. (2016). Juvenile delinquency in Chinese adolescents: An ecological review of the literature. Aggression and Violent Behavior, 31, 26-36. doi: 10.1016/j.avb.2016.06.016

Wolff, K. T., Baglivio, M. T., \& Piquero, A. R. (2017). The relationship between adverse childhood experiences and recidivism in a sample of juvenile offenders in community-based treatment. International Journal of Offender Therapy and Comparative Criminology, 61(11), 1210-1242. doi: 10.1177/0306624X15613992

Submetido em: 10-11-2019

Aceito em: 17-12-2019 